\title{
An Extension of Baum-Fulton-MacPherson's Riemann-Roch Theorem for Singular Varieties
}

\author{
By
}

Shoji YOKURA *

\section{Introduction}

In [1] Baum, Fulton and MacPherson extend the Grothendieck-RiemannRoch theorem (abbr. GRR) for non-singular projective varieties to (possibly singular) quasi-projective varieties over any field and proper morphisms. More precisely, in [1, Chapter 1] they prove the Riemann-Roch (abbr. RR) in the case of (possibly singular) complex projective varieties with the ordinary singular homology theory with rational coefficients, by deformation to the normal bundle, and in [1, Chapter 2], in more general, they prove the $R R$ in the case of (possibly singular) quasi-projective varieties over any field and proper morphisms with the Chow homology theory with rational coefficeints [3], by the "Grassmannian graph construction". If the field is the complex numbers $C$, then the above Chow homology theory can be replaced by the singular homology theory (and Borel-Moore homology theory for non-compact varieties). Thus [1, Chap. 2] generalizes [1, Chap. 1].

In this paper our varieties are complex projective varieties and the homology is the singular homology theory with rational coefficeints.

Let $\boldsymbol{K}_{0}$ be the covariant functor of Grothendieck groups of algebraic coherent sheaves. For a morphism $f: X \rightarrow Y$ the pushforward $f_{*}: K_{0}(X) \rightarrow$ $K_{0}(Y)$ is defined to be the alternating sum of higher direct images $R^{l} f_{*}$. Let $H_{2 *}(; Q)$ be the even part of the $Q$-homology covariant functor. The GRRtheorem says ("homologically") that if $\tau: K_{0}(X) \rightarrow H_{2 *}(X ; Q)$ is defined by $\tau(\mathscr{F}):=\left[\operatorname{td}\left(T_{X}\right) \operatorname{ch}(\mathscr{F})\right] \cap[X]\left(=t d\left(T_{X}\right) \cap(\operatorname{ch}(\mathscr{F}) \cap[X])\right)$, where td is the Todd class and ch is the Chern character, then $\tau$ becomes a natural transformation.

Communicated by M. Kashiwara, September 24, 1992.

1991 Mathematics Subject Classification : 14B05, 14C40, 14F99, 18F99, 57R20

* Department of Mathematics, College of Liberal Arts, University of Kagoshima, Kagoshima 890, Japan 
When it comes to singular varieties, the above definition of $\tau(\mathscr{F})$ does not make any sense since the tangent bundle $T_{X}$ cannot be defined. However, by introducing the localized Chern character $\operatorname{ch}_{X}^{M}(\mathscr{F})$ of the coherent sheaf $\mathscr{F}$ (which is a homology class of $X$ ) with $X$ embedded into a non-singular quasi-projective variety $M$, as a substitute of $(\operatorname{ch}(\mathscr{F}) \cap[X])$, Baum-Fulton-MacPherson proved in [1] that there exists a unique natural transformation $T d_{*}: K_{0} \rightarrow H_{2 *}(; Q)$ satisfying the extra condition that for a smooth variety $X T d_{*}\left(\mathcal{O}_{X}\right)$ is equal to the Poincare dual of the usual total Todd cohomology class $t d\left(T_{X}\right)$ of the tangent bundle $T_{X}$, where $\mathcal{O}_{X}$ is the structure sheaf of $X$. Here for a variety $X \operatorname{Td}_{*}(\mathscr{F})$ is defined by $i^{*} t d\left(T_{M}\right) \cap \operatorname{ch}_{X}^{M}(\mathscr{F})$, which turns out to be independent of the choice of the embedding $i: X \rightarrow M$. For a smooth variety $X i^{*} t d\left(T_{M}\right) \cap \operatorname{ch}_{X}^{M}(\mathscr{F})$ is equal to $\operatorname{td}\left(T_{X}\right) \cap \operatorname{ch}(\mathscr{F}) \cap[X]$, thus in the category of smooth varieties $T d_{*}$ is nothing but the GRR-transformation $\tau$ above.

For a variety $X T d_{*}(X):=T d_{*}\left(\mathcal{O}_{X}\right)$ is called the homology Todd class of $X$, the 0 -th component of which is the arithmetic genus $\chi\left(X, \mathcal{O}_{X}\right)$ of $X$. And $T d_{*}$ is multiplicative, i. e., $T d_{*}(X \times Y)=T d_{*}(X) \times T d_{*}(Y)$, which is a generalization of $\chi\left(X \times Y, \mathcal{O}_{X \times Y}\right)=\chi\left(X, \mathcal{O}_{X}\right) \chi\left(Y, \mathcal{O}_{Y}\right)$. Thus the homology Todd class is a generalization of the arithmetic genus to higher dimensional homology classes. The above Baum-Fulton-MacPherson's Riemann-Roch theorem (abbr. BFM-RR) has another aspect, besides being an extension of GRR, that the natural transformation $T d_{*}: K_{0} \rightarrow H_{2 *}(; Q)$ is a "singular" version of the usual Todd cohomology class of non-singular varieties (cf. MacPherson's survey article [10]). As commented in the introduction of [1], establishing BFM-RR was clearly motivated by the "singular" version of Chern cohomology class, i. e., the Chern-MacPherson homology class [9] : Let $F$ be the constructible function covariant functor. Then there exists a unique natural transformation $C_{*}: \mathbb{F} \rightarrow$ $H_{2 *}(; Z)$ satisfying the extra condition that for a smooth variety $X C_{*}\left(\mathbb{1}_{X}\right)$ is equal to the Poincaré dual of the usual total Chern cohomology class $c\left(T_{X}\right)$ of the tangent bundle $T_{X}$, where $\mathbb{1}_{X}$ is the characteristic function on $X$. For a variety $X$, $C_{*}(X):=C_{*}\left(1_{X}\right)$ is called the Chern-MacPherson class of $X$, the 0 -th component of which is the topological Euler-Poincaré characteristic $\chi(X)$ of $X$. Recently Kwieciński [7] has proved that $C_{*}$ is multipliciative, i. e., $C_{*}(X \times Y)=$ $C_{*}(X) \times C_{*}(Y)$, which is a generalization of $\chi(X \times Y)=\chi(X) \chi(Y)$ (see also $[8]$ ).

In [12] the author extends $C_{*}$ so that the Chern class $c$ in the above extra condition can be replaced by the Chern polynomial $c_{(q)}=\Sigma_{i \geq 0} q^{i} c_{i}$. In this paper, in the same spirit as that in $[12]$, we shall extend the BFM-RR-transformation $T d_{*}$ so that the Todd class $t d$ in the above extra condition can be replaced by the "Todd polynomial" $t d_{(q)}=\Sigma_{i \geq 0} q^{i} t d_{i}$. 
Theorem A. Let $\boldsymbol{K}_{0}^{(q)}(X):=K_{0}(X) \otimes Q\left[q, q^{-1}\right]$ and for a morphism $f: X \rightarrow$ $Y$ the twisted pushforward $f_{*}^{(q)}: \boldsymbol{K}_{0}^{(q)}(X) \rightarrow K_{0}^{(q)}(Y)$ is defined simply by $f_{*}^{(q)}:=$ $q^{\operatorname{dim} X-\operatorname{dim} Y} f_{*}$, where $f_{*}: K_{0}(X) \rightarrow K_{0}(Y)$ is the original one. And let $H_{2 *}^{(q)}(; Q):=$ $H_{2 *}\left(; Q\left[q, q^{-1}\right]\right)$. Then there exists a unique natural transformation $\operatorname{Td}_{(q) *}$ : $K_{0}^{(q)} \rightarrow H_{2 *}^{(q)}(; Q)$ satisfying the extra condition that

$$
T d_{(q) *}\left(\mathcal{O}_{X}\right)=t d_{(q)}\left(T_{X}\right) \cap[X] \text { for smooth } X
$$

and such that if we "evaluate" $T d_{(q) *}$ at $q=1$, then we recover $B F M-R R T d_{(1) *}=$ $T d_{*}$.

To obtain the above "twisted" natural transformation in a similar way to that of the BFM-RR $T d_{*}$, we consider how to modify the basic ingredients $\operatorname{td}\left(T_{M}\right)$ and $\operatorname{ch}_{X}^{M}(\mathscr{F})$ in the BFM-RR-theorem. In BFM-RR $\operatorname{ch}_{X}^{M}(\mathscr{F})$ is defined by $\operatorname{ch}_{X}^{M}(\mathscr{F}):=\operatorname{ch}_{X}^{M}(\mathscr{E}$.$) , where \mathscr{E}$. is a resolution of the sheaf $i_{*} \mathscr{F}$ for an embedding $i: X \rightarrow M$. In our case we define $\operatorname{ch}_{(q) X}^{M}(\mathscr{F}):=q^{\operatorname{dim} X-\operatorname{dim} M} \operatorname{ch}_{(q) X}{ }^{M}(\mathscr{E}$.$) ,$ by introducing some "twisting coefficient", where $c h_{(q)}=\sum_{l \geq 0} q^{i} c h_{l}$ is the "Chern character polynomial". Then, in a similar manner as in [1], we can show that for a coherent sheaf $\mathscr{F}$ and for an embedding $i: X \rightarrow M, i^{*} t d_{(q)}\left(T_{M}\right) \cap$ $\operatorname{ch}_{(q) X}{ }_{M}^{M}(\mathscr{F})$ is actually independent of the embedding $i: X \rightarrow M$ and that the transformation $T d_{(q) *}: K_{0}^{(q)} \rightarrow H_{2 *}^{(q)}(; Q)$ defined by $T d_{(q) *}(\mathscr{F})=i^{*} t d_{(q)}\left(T_{M}\right) \cap$ $\operatorname{ch}_{(q) X}{ }^{M}(\mathscr{F})$ is nothing but the natural transformation in the above theorem.

It turns out that there is a simple relationship between the "twisted" natural transformation $T d_{(q) *}$ and BFM-RR $T d_{*}$ as follows: for a variety $X$ and a coheret sheaf $\mathscr{F}$ on $X$,

$$
T d_{(q) *}(\mathscr{F})=q^{\mathrm{d} m X} \sum_{i \geq 0} q^{-\imath} T d_{*_{\iota}}(\mathscr{F})
$$

where the natural transformation $T d_{*_{i}}: K_{0} \rightarrow H_{2 i}(; Q)$ is the projection of $T d_{*}$ : $K_{0} \rightarrow H_{2 *}(; Q)$ to the $2 i$-dimensional component. If, from the very beginning, we define the "twisted" natural transformation $T d_{(q) *}: K_{0}^{(q)} \rightarrow H_{2 *}^{(q)}(; Q)$ by (0.1) and by extending it linearly with respect to the Laurent polynomial ring $\boldsymbol{Q}\left[q, q^{-1}\right]$, then it is not hard to see Theorem A itself. However, the aim of this paper is to prove Theorem $A$ in an analogous manner to that of BFM-RR Theorem, as a natural extension or generalization of it.

As a corollary of the above theorem, we can get the following "twisted" version of GRR :

Corollary B. For a non-singular projective variety $X$, if we define the homomorphism $\tau^{(q)}: K_{0}^{(q)}(X) \rightarrow H^{2 *(q)}(X ; Q)\left(:=H^{2 *}\left(X ; Q\left[q, q^{-1}\right]\right)\right) b y \tau^{(q)}(\mathscr{F}):=$ 
$\operatorname{ch}_{(q)}(\mathscr{F}) t d_{(q)}\left(T_{X}\right)$ and extending linearly with respect to $Q\left[q, q^{-1}\right]$, then $\tau^{(q)}$ becomes a natural transformation, i. e., for a morphism $f: X \rightarrow Y$ the following equality holds :

$$
c h_{(q)}\left(f_{*}^{(q)} \mathscr{F}\right) t d_{(q)}\left(T_{Y}\right)=f_{*}\left(c h_{(q)}(\mathscr{F}) t d_{(q)}\left(T_{X}\right)\right)
$$

where $f_{*}: H^{2 *(q)}(X ; Q) \rightarrow H^{2 *(q)}(Y ; Q)$ is the Gysin homomorphism.

If we consider ' $\boldsymbol{K}_{0}^{(q)}$ to be just a linear extension of the functor $\boldsymbol{K}_{0}$ with respect to the Laurent polynomial ring $Q\left[q, q^{-1}\right]$, then by considering the map from a smooth $X$ to a point we can see that one cannot get such a natural transformation $\tau:{ }^{\prime} K_{0}^{(q)} \rightarrow H_{2 *}^{(q)}(; Q)$ satisfying the condition that $\tau\left(\mathcal{O}_{X}\right)=$ $t d_{(q)}\left(T_{X}\right) \cap[X]$ for smooth $X$. In general we can show

Theorem C. Let $c l: \mathbb{K} \rightarrow H^{2 *}\left(; Q\left[q, q^{-1}\right]\right)$ be a total characteristic class of complex vector bundles. Then $\tau:{ }^{\prime} K_{0}^{(q)} \rightarrow H_{2 *}^{(q)}(; Q)$ is a natural transformation satisying that $\tau\left(\mathcal{O}_{X}\right)=c l\left(T_{X}\right) \cap[X]$ for smooth $X$ if and only if $c l=\lambda \circ$ td and $\tau$ $=\lambda \circ T d_{*}$ for some $\lambda \in Q\left[q, q^{-1}\right]$.

The organization of the paper is as follows. In $\S 1$ we recall some basic facts and results about characteristic cohomology classes. In $\S 2$ we show the universality theorem of $\mathrm{BFM}-\mathrm{RR}$ transformation $T d_{*}$ and Theorem $\mathrm{C}$. In $\S 3$ we prove Theorem $A$ and give a characterization of the "twisted" $B F M-\mathbb{R} R$ transformation $T d_{(q) *}$.

\section{§1. Preliminaries}

In this section we introduce some notation and recall some basic results on characteristic classes of complex vector bundles, which we will use in the rest of the paper.

A usual characteristic class $c l$ of complex vector bundles is a rule assigning to any complex vector bundle $E$ over any topological space $X$ an element $c l(E)$ of the cohomology group of $X$ such that it satisfies the naturality condition, i. e., $\operatorname{cl}\left(f^{*} E\right)=f^{*} \operatorname{cl}(E)$ for any map $f: Y \rightarrow X$. To paraphrase this more fashionably, let $\mathscr{V}_{\text {ect }}: \mathscr{T}_{0} \not \rightarrow \mathscr{E} n s$ be the contravariant functor from the category $\mathscr{T}_{0} \not$ of topological spaces to the category $\mathscr{E} n s$ of sets, such that $\mathscr{V}_{\text {ect }}(X)=$ the set of isomorphism classes of vector bundles over $X$, and let $H^{*}(; \Lambda):=H^{*}(; \mathbb{Z})_{\mathbb{Z}}$ $\Lambda: \mathscr{T}_{0} p \rightarrow \mathscr{E} n s$ be the usual cohomology contravariant functor, where we ignore the algebraic structures of cohomology rings. Then the above characteristic class $c l$ is a natural transformation $c l: \mathscr{V}_{e c t} \rightarrow H^{*}(; \Lambda)$. If $c l$ is multiplicative, i. e., 
$c l(E \oplus F)=c l(E) c l(F)$, then $c l$ can be strengthened to be a natural transformation from the Grothendieck contravariant functor $\boldsymbol{K}^{0}$ to $H^{*}(; \Lambda)$, where $\boldsymbol{K}^{0}$ and $H^{*}(; \Lambda)$ are now functors from $\mathscr{T}_{0} \not p$ to the category $\mathscr{R} i n g$ of rings.

Let $n$ be a non-negative integer and a partition of $n$ be a non-decreasing sequence $\left\{r_{1}, r_{2}, \ldots, r_{k}\right\}$ of positive integers whose sum is exactly equal to $n$. The (only) partition of zero is conventionally zero itself. Let $I_{k}(n)$ denote such a partition $\left\{r_{1}, r_{2}, \ldots, r_{k}\right\}$ of $n$ and let $I(n)$ denote the set of all partitions of $n$ and $p(n)$ denote the cardinality $|I(n)|$ of the set $I(n)$. Let $P_{n}\left(c_{1}, c_{2}, \ldots, c_{n}\right)$ be a homogeneous polynomial of degree $n$ with the weight of $c_{i}$ being $i$. Thus $P_{n}\left(c_{1}, c_{2}, \ldots, c_{n}\right)$ is a linear combination of $I_{k}(n)$-Chern class $c_{I_{k}(n)}:=c_{r_{1}} c_{r_{2}} \ldots$ $c_{r_{k}}$, i. e.,

$$
P_{n}\left(c_{1}, c_{2}, \ldots, c_{n}\right)=\sum_{I_{k}(n) \in J(n)} \lambda_{I_{k}(n)} c_{I_{k}(n)} .
$$

Any characteristic class $c l: \mathscr{V}_{e c t} \rightarrow H^{*}(; \Lambda)$ can be expressed as a formal power series of Chern classes, i. e., $c l=\lambda_{0} c_{0}+\sum_{n \geq 1} P_{n}\left(c_{1}, c_{2}, \ldots, c_{n}\right)$. A fundamental result about Chern classes is that the total Chern class $c: K^{0} \rightarrow H^{2 *}(; \Lambda)^{\wedge}:=1+$ $\sum_{i \geq 1} H^{2 i}(; \Lambda)$ is universal for all multiplicative characteistic classes. I. e., given any multiplicative characteristic class $c l: K^{0} \rightarrow H^{2 *}(; \Lambda)^{\wedge}$, there exists an endomorphism $\Phi_{c l}: H^{2 *}(; \Lambda)^{\wedge} \rightarrow H^{2 *}(; \Lambda)^{\wedge}$ such that the following diagram commutes :

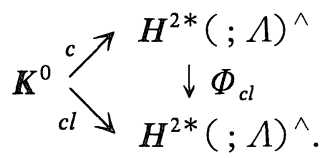

Indeed there exists a unique multiplicative sequence $M:=\left\{1, M_{1}\left(x_{1}\right), M_{2}\left(x_{1}\right.\right.$, $\left.\left.x_{2}\right), \ldots, M_{i}\left(x_{1}, x_{2}, \ldots, x_{2}\right), \ldots\right\}$ such that $c l=1+\sum_{n \geq 1} M_{n}\left(c_{1}, c_{2}, \ldots, c_{n}\right)$. Thus the multiplicative sequence $M$ gives rise to the endomorphism $\Phi_{c l}$ : $H^{2 *}(; \Lambda)^{\wedge} \rightarrow H^{2 *}(; \Lambda)^{\wedge}$ defined by

$$
\Phi_{c l}\left(1+\Sigma_{i \geq 1} x_{\nu}\right):=1+\sum_{i \geq 1} M_{i}\left(x_{1}, x_{2}, \ldots, x_{\nu}\right)
$$

and $c l$ is the composite of the total Chern class $c=1+\sum_{i \geq 1} c_{i}$ and the endomorphism $\Phi_{c l}$.

Let $X$ be a compact complex manifold of dimension $m \geq n$ and then as usual we define the $2 n$-dimensional characteristic cohomology class $c_{I_{k}(n)}(X)$ of the manifold $X$ as follows :

$$
c_{I_{k}(n)}(X):=c_{I_{k}(n)}\left(T_{X}\right)=c_{r_{1}}\left(T_{X}\right) c_{r_{2}}\left(T_{X}\right) \ldots c_{r_{k}}\left(T_{X}\right),
$$


where $T_{X}$ is the tangent bundle of $X$. And we denote the $2(m-n)$-dimensional homology class $c_{I_{k}(n)}(X) \cap[X]$ by simply $c_{I_{k}(n)}[X]$. In particular, if $m=n$, then $c_{I_{k}(n)}[X]$ is an integer and called the $I_{k}(n)$-Chern number of $X$ (see [11, $\S 16])$.

For a partition $I_{j}(n)=\left\{r_{1}, r_{2}, \ldots, r_{j}\right\}$ of $n$ we define the $I_{j}(n)$-projective space $\boldsymbol{P}^{I^{(n)}}$ by :

$$
\boldsymbol{P}^{I_{j}^{(n)}}:=\boldsymbol{P}^{r_{1}} \times \boldsymbol{P}^{r_{2}} \times \ldots \times \boldsymbol{P}^{r_{j}}
$$

With these definitions above, we have the following fundamental result :

Theorem (1.2) ([11, Theorem 16.7 and a remark right after it $]$ ). The $p(n) \times p(n)$ matrix $M_{n}$ whose entries are $I_{k}(n)$-Chern numbers of $I_{j}(n)$-projective spaces $\boldsymbol{P}^{I^{(n)}}$ :

$$
\boldsymbol{M}_{n}:=\left(c_{I_{k}(n)}\left[\boldsymbol{P}^{I_{j}^{(n)}}\right]\right)
$$

is non-singular.

Corollary (1.3) (The linear independence of Chern numbers $[11, \S 16]$ ). If $\left(P_{n}\left(c_{1}, c_{2}, \ldots, c_{n}\right)\right)\left[\boldsymbol{P}^{I_{j}(n)}\right]=0$ for all $I_{j}(n) \in I(n)$, then $P_{n}\left(c_{1}, c_{2}, \ldots, c_{n}\right)=$ 0 as a polynomial. Hence, if $\left(P_{n}\left(c_{1}, c_{2}, \ldots, c_{n}\right)\right)[X]=0$ for any compact complex manifold of dimension $n$, equivalently $\left(P_{n}\left(c_{1}, c_{2}, \ldots, c_{n}\right)\right)\left[T_{X}\right]=0$ by the Poincaré duality, then $P_{n}\left(c_{1}, c_{2}, \ldots, c_{n}\right)=0$ as a polynomial.

Corollary (1.4). If $r$ is a rational number and $\left(\boldsymbol{P}_{n}\left(c_{1}, c_{2}, \ldots, c_{n}\right)\right)\left[\boldsymbol{P}^{I_{j}(n)}\right]$ $=r$ for all $I_{j}(n) \in I(n)$, then $P_{n}\left(c_{1}, c_{2}, \ldots, c_{n}\right)=r \circ t d_{n}\left(c_{1}, c_{2}, \ldots, c_{n}\right)$, where $t d_{n}\left(c_{1}, c_{2}, \ldots, c_{n}\right)$ is the $n$-th Todd class.

Proof. Use the fact (see $[6])$ that $\left(t d_{n}\left(c_{1}, c_{2}, \ldots, c^{n}\right)\right)\left[\boldsymbol{P}^{I_{j}(n)}\right]=1$ for any $I_{j}(n) \in I(n)$ and Corollary (1.3).

Proposition (1.5). ([14]) Let $m \geq n$ and let $X$ and $Y$ be compact complex manifolds of dimensions $m-n$ and $n$, respectively, and let $\pi: X \times Y \rightarrow X$ be the projection. Then

$$
\pi_{*}\left(c_{I_{k}(n)}[X \times Y]\right)=\left(c_{I_{k}(n)}[Y]\right)[X] .
$$

Corollary (1.6). Let $m \geq n$ and let $X$ and $Y$ be compact complex manifolds of dimensions $m-n$ and $n$, respectively, and let $\pi: X \times Y \rightarrow X$ be the projection. Then 


$$
\pi_{*}\left(P_{n}\left(c_{1}, c_{2}, \ldots, c_{n}\right)[X \times Y]\right)=\left(P_{n}\left(c_{1}, c_{2}, \ldots, c_{n}\right)[Y]\right)[X]
$$

We introduce more terminology. The natural transformation $c l=\Sigma_{i \geq 0} c l_{i}$ : $\mathscr{V}$ ect $\rightarrow H^{*}(; \Lambda)$ shall be called a total characteristic class, instead of just a characteristic class. Here $c l_{0}=\lambda_{0} c_{0}$ and $c l_{i}=P_{i}\left(c_{1}, c_{2}, \ldots, c_{i}\right)$. If we are arbitrarily given isobaric polynomials $c l_{0}=\lambda_{0} c_{0}$ and $c l_{i}=P_{i}\left(c_{1}, c_{2}, \ldots, c_{i}\right)(1 \leq i \leq n)$, then $c l^{(n)}:=\sum_{0 \leq i \leq n} c l_{i}$ is called a degree- $n$ characteristic class.

Using Corollary (1.6) we can show the following proposition, which is a generalization of the above "linear independence of Chern numbers":

Proposition (1.7) ([14]). Let $\mathrm{cl}^{(n)}$ be a degree-n characteristic class. If $c l^{(n)}\left(T_{X}\right)=0$ for any compact complex manifold of dimension $n$, then $c l^{(n)}=0$ as a polynomial.

\section{§ 2. The Universality of BFM-Riemann-Roch Transformation $T d_{*}$}

The uniqueness of the BFM-RR-transformation $T d_{*}: K_{0} \rightarrow H_{2 *}(; Q)$ satisfying the extra condition that $\tau\left(\mathcal{O}_{X}\right)=t d\left(T_{X}\right) \cap\left[X_{\lrcorner}\right.$for any smooth variety $X$ follows from the following lemma, which can be shown by resolution of singularities.

Lemma (2.1) (cf. $[2, \S 4.2]$ ). Let $\tau, \tau^{\prime}: K_{0} \rightarrow H_{2 *}(; Q)$ be two natural transformations. Then $\tau=\tau^{\prime}$ if and only if $\tau\left(\mathcal{O}_{W}\right)=\tau^{\prime}\left(\mathcal{O}_{W}\right)$ for any smooth variety $W$.

Proof. It suffices to note that for any variety $X K_{0}(X)$ is generated by the structure sheaves $\mathcal{O}_{V}$ for all subvarieties $V$ of $X$, and furthermore that for any structure sheaf $\mathcal{O}_{V}$ there exist a finitely many smooth varietes $W_{i}$, morphisms $\pi_{i}: W_{i} \rightarrow V$ and some integers $m_{i}$ 's such that

$$
\mathcal{O}_{V}=\Sigma_{i} m_{i} \pi_{i *} \mathcal{O}_{w_{i}}
$$

This lemma is an analogue of : For two natural transformations $\tau, \tau^{\prime}: \boldsymbol{F} \rightarrow$ $H_{2 *}(; Z) \tau=\tau^{\prime}$ if and only if $\tau\left(1_{X}\right)=\tau^{\prime}\left(1_{X}\right)$ for any smooth variety $X$. The proof of this is similar to that of the above $[5,9]$.

In [1], however, the uniqueness of $T d_{*}$ is not shown by using resolution of singularities at all, but by the following strengthened "uniqueness theorem" of $T d_{*}:$ 
"Uniqueness theorem" ([1]). BFM-RR-transformation $\quad T d_{*}: \mathbb{K}_{0} \rightarrow$ $H_{2 *}(; Q)$ is the only natural transformation $\tau$ satisfying the property that

$$
\tau\left(\mathcal{O}_{P^{n}}\right)=\left[\mathbb{P}^{n}\right]+\text { homology classes of lower degrees }
$$

for each projective space $\mathbb{P}^{n}, n=0,1,2, \ldots$.

This "Uniqueness theorem" follows from the fact [1, Chap. III, § 1] that for each variety $X T d_{*} \otimes Q$ induces an isomorphism $K_{0}(X) \otimes Q \stackrel{\sim}{\rightarrow} H_{2 *}(X ; Q)$ and the following "Identity theorem" $([3, \S 5])$ :

"Identity theorem" ([3, § 5]). If $\alpha: H_{2 *}(; Q) \rightarrow H_{2 *}(; Q)$ is a natural transformation such that for each projective space $\mathbb{P}^{i}, i=0,1,2,3, \ldots$,

$$
\alpha\left(\left[\mathbb{P}^{i}\right]\right)=\left[\mathbb{P}^{i}\right]+\text { homology classes of lower degrees, }
$$

then $\alpha$ must be the identity.

It turns out that "Uniqueness theorem" tells us more about BFM- $R \mathbf{R}-$ transformation $T d_{*}$ :

Theorem (2.2) (The universality of BFM-RR-transformation $T d_{*}[15$, Theorem 1]). If $\tau: \mathbb{K}_{0} \rightarrow H_{2 *}(; Q)$ is a natural transformation, then there exists a unique sequence $\left\{r_{i}\right\}_{i \geq 0}$ of rational numbers such that $\tau=\sum_{i \geq 0} r_{i} T d_{* i}$, where $T d_{* i}: H_{2 *}(; Q) \rightarrow H_{2 i}(; Q)$ is the projection of $T d_{*}$ to the $2 i$-dimensional component. Thus BFM-RR-transformation $T d_{*}: \mathbb{K}_{0} \rightarrow H_{2 *}(; Q)$ is universal in the sense that for any natural transformation $\tau: \mathbb{K}_{0} \rightarrow H_{2 *}(; Q)$ there exists a unique natural transformation $\Phi_{\tau}: H_{2 *}(; Q) \rightarrow H_{2 *}(; Q)$ such that $\tau=\Phi_{\tau} \circ T d_{*}$, where $\Phi_{\tau}$ is defined by $\Phi_{\tau}\left(\sum_{i \geq 0} x_{i}\right)=\sum_{l \geq 0} r_{i} x_{i}$.

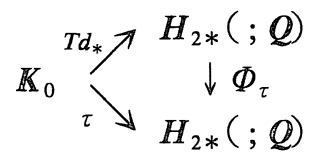

By the same argument (see [15, Proof of Theorem 1]) and using "Identity theorem", we can show the following

Theorem (2.3) (The linearity of endomorphism $H_{2 *}(; \mathbb{Q}) \rightarrow H_{2 *}(; \mathbb{Q})$ ). If $\tau: H_{2 *}(; \mathbf{Q}) \rightarrow H_{2 *}(; \mathbb{Q})$ is a natural transformation, then there exists a unique sequence $\left\{r_{i}\right\}_{i \geq 0}$ of rational numbers such that $\tau=\sum_{i \geq 0} r_{i} \pi_{i}$, where $\pi_{i}: H_{2 *}(; Q)$ $\rightarrow H_{2 i}(; \mathbb{Q})$ is the projection to the $2 i$-dimensional component. Namely, $\tau\left(\sum_{i \geq 0} x_{i}\right)$ $=\sum_{i \geq 0} r_{i} x_{i}$. 
Remark (2.4). It is easy to see that "Uniqueness theorem" and Theorem (2.2) are equivalent and that "Identity theorem" and Theorem (2.3) are equivalent.

Remark (2.5). Establishing Theorem (2.2) has been motivated by Kennedy's conjecture that any natural transformation $\tau: F \rightarrow H_{2 *}(; Z)$ can be uniquely expressed as $\tau=\Sigma_{i \geq 0} m_{i} C_{*_{i}}$, where $C_{*_{i}}: H_{2 *}(; Z) \rightarrow H_{2 i}(; Z)$ is the projection of the natural transformation $C_{*}$ to the $2 i$-dimensional component and each $m_{i}$ is an integer. This conjecture is still unsolved. Note that Kennedy's conjecture is equivalent to claiming that $C_{*}: F \rightarrow H_{2 *}(; Z)$ is the unique natural transformation satisfying the property that

$$
\tau\left(1_{P^{n}}\right)=\left[\boldsymbol{P}^{n}\right]+\text { homology classes of lower degrees }
$$

for each projective spdce $\boldsymbol{P}^{n}, n=0,1,2, \ldots$

The "linear" natural transformation $\sum_{l \geq 0} r_{i} T d_{* i}$ has the following "characterization" :

Theorem (2.6). Let $c l^{(n)}: \mathscr{V}_{\text {ect }} \rightarrow H^{2 *}(; Q)$ be a degree-n characteristic class of complex vector bundles, and let $\left\{c l^{(n)}\right\}_{n \geq 0}$ be a sequence of degree- $n$ characteristic classes. Then $\tau: K_{0} \rightarrow H_{2 *}(; Q)$ is a natural transformation satisfying the "dimension-wise universal smooth condition" that $\tau\left(\mathcal{O}_{X}\right)=\mathrm{cl}^{(\mathrm{dim} X)}\left(T_{X}\right) \cap[X]$ for any smooth variety $X$, if and only if there exists a unique sequence $\left\{r_{i}\right\}_{i \geq 0}$ of rational numbers such that

$$
\begin{gathered}
\tau=\sum_{i \geq 0} r_{i} T d_{* i}, \text { and } \\
c l^{(n)}=\sum_{0 \leq i \leq n} r_{i} t d_{n-\imath} .
\end{gathered}
$$

This theorem follows from Theorem (2.2) and Proposition (1.7), or without using "Uniqueness theorem", we can also show this theorem by Lemma ( 2 . 1), Corollaries (1.4) and (1.6).

Proof I (Using Theorem (2. 2)). By Theorem (2.2) there exists a unique sequence $\left\{r_{i}\right\}_{i \geq 0}$ of rational numbers such that $\tau=\sum_{i \geq 0} r_{i} T d_{*_{i}}$. So it remains to prove that each degree- $n$ characteristic class $c l^{(n)}=\sum_{0 \leq i \leq n} r_{i} t d_{n-\imath}$. Since $\left(\sum_{i \geq 0} r_{i} T d_{*_{i}}\right)\left(\mathcal{O}_{X}\right)=\left(\sum_{0 \leq i \leq n} r_{i} t d_{n-i}\right)\left(T_{X}\right)$ for any smooth variety $X$, we have the following equality :

$$
c l^{(n)}\left(T_{X}\right) \cap[X]=\left(\sum_{0 \leq i \leq n} r_{i} t d_{n-i}\right)\left(T_{X}\right) \cap[X]
$$


for any smooth variety of dimension $n$, i. e., by the Poincaré duality, we have

$$
c l^{(n)}\left(T_{X}\right)=\left(\sum_{0 \leq i \leq n} r_{i} t d_{n-i}\right)\left(T_{X}\right)
$$

for any smooth variety of dimension $n$. i. e.,

$$
\left(c l^{(n)}-\sum_{0 \leq i \leq n} r_{i} t d_{n-i}\right)\left(T_{X}\right)=0
$$

for any smooth variety of dimension $n$. Thus from Proposition (1.7) we can conclude that $c l^{(n)}-\sum_{0 \leq i \leq n} r_{i} t d_{n-i}=0$, i. e.,

$$
c l^{(n)}=\sum_{0 \leq i \leq n} r_{i} t d_{n-i} .
$$

Q. E. D.

Proof II (Using Lemma (2. 1)). Suppose that $\tau: K_{0} \rightarrow H_{2 *}(; \boldsymbol{Q})$ is a natural transformation satisfying the "dimension-wise universal smooth condition" that $\tau\left(\mathcal{O}_{X}\right)=c l^{(\operatorname{dim} X)}\left(T_{X}\right) \cap[X]$ for any smooth variety $X$, with $c l^{(0)}=\lambda_{0}^{0} c_{0}$ and $c l^{(n)}=\lambda_{0}^{n}+\sum_{1 \leq i \leq n} P_{i}^{n}\left(c_{1}, c_{2}, \ldots, c_{i}\right)$. For each partition $I_{j}(i)$ of $i$, we consider the projection $\pi: \boldsymbol{P}^{n-i} \times \boldsymbol{P}^{I_{j}(i)} \rightarrow \boldsymbol{P}^{n-i}$. Then by the naturality of our transformation $\tau$, since $\pi_{*}\left(\mathcal{O}_{\boldsymbol{P}^{n-i} \times \boldsymbol{P}^{I_{j}(i)}}\right)=\chi\left(\boldsymbol{P}^{I_{j}^{(i)}}, \mathcal{O}_{\boldsymbol{P}_{j} I^{(i)}}\right) \mathcal{O}_{\boldsymbol{P}^{n-i}}=\mathcal{O}_{\boldsymbol{P}^{n-i}}$ (because $\chi\left(\boldsymbol{P}^{I^{j}}{ }^{(i)}\right.$, $\left.\mathcal{O}_{\boldsymbol{P}} I_{j}(i)\right)=1$, see $\left.[6]\right)$, we have

$$
\pi_{*} \tau\left(\mathcal{O}_{\boldsymbol{P}^{n-i} \times \boldsymbol{P}_{j} I_{j}^{(i)}}\right)=\tau\left(\mathcal{O}_{\boldsymbol{P}^{n-i}}\right)
$$

Hence by the "dimension-wise universal smooth condition", we have

$$
\pi_{*}\left(c l^{(n)}\left[\boldsymbol{P}^{n-i} \times \boldsymbol{P}^{I_{j}(i)}\right]\right)=c l^{(n-i)}\left[\boldsymbol{P}^{n-i}\right] .
$$

Therefore, by Corollary (1.6) we get

$$
\begin{aligned}
\text { LHS. of }(* *)= & \pi_{*}\left(\cdots+\boldsymbol{P}_{i}^{n}\left(c_{1}, c_{2}, \ldots, c_{i}\right)\left[\boldsymbol{P}^{n-i} \times \boldsymbol{P}^{I_{j}(i)}\right]+\cdots\right) \\
= & \left(\boldsymbol{P}_{i}^{n}\left(c_{1}, c_{2}, \ldots, c_{i}\right)\left[\boldsymbol{P}^{I_{j}(i)}\right]\right)\left[\boldsymbol{P}^{n-i}\right] \\
& + \text { homology classes of degree }<2(n-i) .
\end{aligned}
$$

RHS. of $(* *)=\lambda_{0}^{n-i}\left[\boldsymbol{P}^{n-i}\right]+$ homology classes of degree $<2(n-i)$.

Hence by looking at the $2(n-i)$-dimensional part of $(* *)$, we have the following equality :

$$
\left(\boldsymbol{P}_{i}^{n}\left(c_{1}, c_{2}, \ldots, c_{i}\right)\left[\boldsymbol{P}^{I_{j}(i)}\right]\right)\left[\boldsymbol{P}^{n-i}\right]=\lambda_{0}^{n-i}\left[\boldsymbol{P}^{n-i}\right] \text {, i. e., }
$$




$$
P_{i}^{n}\left(c_{1}, c_{2}, \ldots, c_{l}\right)\left[P^{I_{j}^{(l)}}\right]=\lambda_{0}^{n-i} \text { for each } I_{J}(i) \in I(i) .
$$

Hence by Corollary (1.4) we can conclude that

$$
P_{i}^{n}\left(c_{1}, c_{2}, \ldots, c_{i}\right)=\lambda_{0}^{n-l} t d_{i}\left(c_{1}, c_{2}, \ldots, c_{i}\right)
$$

Thus, letting $r_{i}=\lambda_{0}^{i}$, we can see that there exists a unique seqence $\left\{\boldsymbol{r}_{i}\right\}_{i \geq 0}$ of rational numbers such that $c l^{(n)}=\sum_{0 \leq i \leq n} r_{i} t d_{n-i}$ for each $n$. Then it remains to show that $\tau=\sum_{i \geq 0} r_{i} T d_{*_{i}}$. Since

$$
\begin{aligned}
\tau\left(\mathcal{O}_{X}\right)=c l^{(\operatorname{dim} X)}\left(T_{X}\right) \cap[X] & =\left(\sum_{0 \leq i \leq n} r_{i} t d_{n-i}\right)\left(T_{X}\right) \cap[X] \\
& =\left(\sum_{i \geq 0} r_{i} T d_{*_{l}}\right)\left(\mathcal{O}_{X}\right),
\end{aligned}
$$

it follows from Lemma (2.1) that $\tau=\sum_{i \geq 0} r_{i} T d_{*_{l}}$.

Q. E. D.

Let $\Lambda$ be a commutative integral domain with unit and let ' $\boldsymbol{K}_{0}^{\Lambda}:=\boldsymbol{K}_{0}(\quad) \otimes$ $\Lambda$ be just the linear extension of the functor $\boldsymbol{K}_{0}$ with respect to $\Lambda$. Then as a corollary of Proof II, we can show the following

Corollary (2.7). Let $c l: \mathscr{V}$ ect $\rightarrow H^{2 *}(; \Lambda)$ be a total characteristic class of complex vector bundles. Then $\tau:{ }^{\prime} K_{0}^{\Lambda} \rightarrow H_{2 *}(; \Lambda)$ is a natural transformation satisfying the extra condition that for a smooth $X \tau\left(\mathcal{O}_{X}\right)=\operatorname{cl}\left(T_{X}\right) \cap[X]$, if and only if there exists an element $\lambda$ of $\Lambda$ such that $c l=\lambda \circ t d$ and $\tau=\lambda \circ T d_{*}$.

Thus it follows from Corollary (2.7) that there is no natural transformation $\tau:{ }^{\prime} K_{0}^{(q)} \rightarrow H_{*}^{(q)}(; Q)$ satisfying the extra condition that $\tau\left(\mathcal{O}_{X}\right)=t d_{(q)}\left(T_{X}\right) \cap$ $[X]$ for any smooth $X$. A similar situation occurs in $C_{*}$ and in [12] we extended $C_{*}$ to the Chern polynomial $c_{(q)}=1+\sum_{i \geq 1} q^{i} c_{1}$, introducing the "twisted" constructible function functor. In the following section we will discuss the extension of BFM-RR $T d_{*}$ to the Todd polynomial $t d_{(q)}=1+\sum_{i \geq 1} q^{i} t d_{i}$.

\section{§ 3. A "Twisted" Version $T d_{(q) *}$ of Baum-Fulton- MacPherson-Riemann-Roch $\boldsymbol{T d}_{*}$}

First we recall the Chern character polynomial $c h_{(q)}$ (this notation and definition are borrowed from Hirzebruch's book $[6, \S 12]$ ) and the Todd polynomial $t d_{(q)}$.

Definition (3.1). Let $q$ be an indeterminant.

(i) Chern character polynomial $c h_{(q)}$ is defined by : 


$$
c h_{(q)}:=c h_{0}+\sum_{i \geq 1} q^{i} c h_{i}
$$

where $c h=c h_{0}+\sum_{i \geq 1} c h_{i}$ is the total Chern character. To be more precise, if we let $\alpha_{i}$ 's be Chern roots of $E$,

$$
c h_{(q)}(E):=\sum_{1 \leq i \leq \operatorname{rank} E} \exp \left(q \alpha_{i}\right)
$$

(ii) Todd polynomial $t d_{(q)}$ is defined by :

$$
t d_{(q)}:=1+\sum_{i \geq 1} q^{i} t d_{i}
$$

where $t d=1+\sum_{i \geq 1} t d_{i}$ is the total Todd class. To be more precise,

$$
t d_{(q)}(E):=\prod_{1 \leq i \leq \mathrm{rank} E}\left\{q \alpha_{i} /\left(1-\exp \left(-q \alpha_{i}\right)\right\}\right.
$$

It is well-known (e. g., see [4] or [6]) that an important connection between the usual Chern character $c h=c h_{(1)}$ and Todd class $t d=t d_{(1)}$ is the following formula, which plays a key role in the formulation of GRR and BFMRR :

Formula (3.2). $\quad \sum_{0 \leq p \leq n}(-1)^{p} \operatorname{ch}\left(\Lambda^{p} E^{\vee}\right)=c_{n}(E) t d(E)^{-1}$, where $E$ is a complex vector bundle of rank $n$ and $E^{\vee}$ is the dual of $E$.

The proof of Formula (3.2) is well-known or standard, but we recall it here for the sake of Formula (3.2) $)^{(q)}$ below : Let $\alpha_{i}$ 's be Chern roots of $E(1 \leq i \leq n)$. Then the proof is as follows.

$$
\begin{aligned}
\sum_{0 \leq p \leq n}(-1)^{p} \operatorname{ch}\left(\Lambda^{p} E^{\vee}\right) & =\Sigma_{0 \leq p \leq n}(-1)^{p} \sum_{i_{1}<\ldots<i_{p}} \exp \left(-\alpha_{i_{1}}-\cdots-\alpha_{i p}\right) \\
& =\prod_{1 \leq i \leq n}\left(1-\exp \left(-\alpha_{i}\right)\right) \\
& =\alpha_{1} \ldots \alpha_{n} \prod_{1 \leq i \leq n}\left(\left(1-\exp \left(-\alpha_{i}\right)\right) / \alpha_{i}\right) \\
& =c_{n}(E) t d(E)^{-1}
\end{aligned}
$$

If we follow this proof, i. e., if we replace $\alpha_{i}$ by $q \alpha_{i}$ in the above proof, then we get the following

$$
\text { Formula }(3.2)^{(\mathbf{q})} . \quad \sum_{0 \leq p \leq n}(-1)^{p} c h_{(q)}\left(\Lambda^{p} E^{\vee}\right)=q^{n} c_{n}(E) t d_{(q)}(E)^{-1}
$$

(or $\sum_{0 \leq p \leq n}(-1)^{p} c h_{(q)}\left(\Lambda^{p} E^{\vee}\right)=\left\{c_{(q)}\right\}_{n}(E) t d_{(q)}(E)^{-1}$, if we use the Chern polynomial $c_{(q)}$.) 


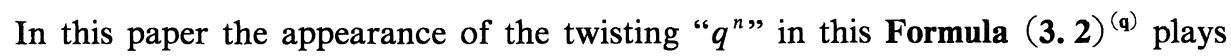
a key role.

Remark (3.3). In [6] Hirzebruch defined a generalized Todd genus or $T_{y}-$ genus as follows.

$$
T_{y}(E):=\prod_{1 \leq i \leq \mathrm{rank} E}\left\{\left((y+1) \alpha_{i} /\left(1-\exp \left(-(y+1) \alpha_{i}\right)\right)-y \alpha_{l}\right\} .\right.
$$

For $E=T_{M}$, the tangent bundle of a complex manifold $M$, if we "evaluate" $T_{y}\left(T_{M}\right)$ at some special values, then we get some known invariants of $M$; e. g., $T_{0}\left(T_{M}\right)$ is the Todd class of $M, T_{-1}\left(T_{M}\right)$ is the Euler-Poincare characteristic of $M$, and $T_{1}\left(T_{M}\right)$ is the signature of $M$. In our definition we drop the additional term " $y \alpha_{\imath}$ ”. If we use Hirzebruch's $T_{y}$-genus, then we have

$$
\sum_{0 \leq p \leq n}(-1)^{p} \operatorname{ch}_{(q)}\left(\Lambda^{p} E^{\vee}\right)=c_{n}(E) T_{(q-1)}(E)^{-1} \sum_{0 \leq p \leq n}(q-1)^{p} c h_{(q)}\left(\Lambda^{p} E^{\vee}\right) .
$$

Indeed,

$$
\begin{aligned}
& \sum_{0 \leq p \leq n}(-1)^{p} c h_{(q)}\left(\Lambda^{p} E^{\vee}\right) \\
&= \prod_{1 \leq i \leq n}\left(1-\exp \left(-q \alpha_{\imath}\right)\right) \\
&= \prod_{1 \leq l \leq n}\left\{\left(1-\exp \left(-q \alpha_{\imath}\right)\right) /\left(\alpha_{\imath}+\alpha_{\imath}(q-1) \exp \left(-q \alpha_{\imath}\right)\right)\right\} \\
& \times \prod_{1 \leq l \leq n}\left(\alpha_{i}+\alpha_{\imath}(q-1) \exp \left(-q \alpha_{\imath}\right)\right) \\
&= \alpha_{1} \ldots \alpha_{n \leq}\left[\prod_{1 \leq l \leq n}\left\{\left(q \alpha_{i} /\left(1-\exp \left(-q \alpha_{\imath}\right)\right)\right)-\alpha_{i}(q-1)\right\}\right]^{-1} \\
& \quad \times \prod_{1 \leq i \leq n}\left(1+(q-1) \exp \left(-q \alpha_{i}\right)\right) \\
&=c_{n}(E) T_{(q-1)}(E)^{-1} \sum_{0 \leq p \leq n}(q-1)^{p} c h_{(q)}\left(\Lambda^{p} E^{\vee}\right) .
\end{aligned}
$$

Thus this formula becomes sort of "redundant", this is why we take up the simpler form $t d_{(q)}$. We do not know whether one can formulate a GRR type theorem for $T_{y}-$ genus or not, which remains to be seen.

Definition (3.4) ( $A$ "twisted" version $\boldsymbol{K}_{0}^{(q)}$ of the Grothendieck covariant functor $\left.K_{0}\right)$. Let $K_{0}^{(q)}(X):=K_{0}(X) \otimes Q\left[q, q^{-1}\right]$ for any variety $X$ and for a morphism $f: X \rightarrow Y$, the pushforward $f_{*}^{(q)}$ is defined by :

$$
f_{*}^{(q)}=q^{\text {reldm }(f)} f_{*},
$$


where $f_{*}$ is the pushforward for the original functor $\boldsymbol{K}_{0}$ and $\operatorname{reldim}(f):=\operatorname{dim} X$ $-\operatorname{dim} Y$ is the relative dimension of $f$. (Then it is obvious that $\boldsymbol{K}_{0}^{(q)}$ is a covaiant functor.)

There are several proofs of BFM-RR $T d_{*}$, i. e., by deformation to the normal bundle $[1$, Chap. 1$]$, by Grassmannian-graph [1, Chap. 2], by using topological $\mathrm{K}$-theory [2], and by Fulton-MacPherson's bivariant theory [4, Chap. 18]. In this paper we follow the proof by deformation to the normal bundle, since it is least hard to give an analogous proof of our "twisted" version $T d_{(q) *}$. Instead of writing down all ingredients and details developed in [1, Chap. 1], we just write down necessary points and key formulae to get our "twisted" version.

The main ingredient in proving BFM-RR-theorem is the localized Chern character $\operatorname{ch}_{X}^{M}\left(E\right.$.), living in the $Q$-homology $H_{2 *}(X ; Q)$, where $X$ is a compact complex subspace of a complex manifold $M$, and $E$. is a complex of topological vector bundles on $M$ which is exact off $X$. To be more precise (for more details see [1, Chap. 1]), $\operatorname{ch}_{X}^{M}(E$.$) is defined as follows : Let d(E.) \in K^{0}(M, M-X)$ be the difference-bundle of the complex $E$. ch: $K^{0}(M, M-X) \rightarrow H^{2 *}(M, M-X$; $Q)$ be the Chern character and $L: H^{2 *}(M, M-X ; Q) \rightarrow H_{2 *}(X ; Q)$ the Lefschetz duality isomorphism. Then $c h_{X}^{M}(E$.$) is defined to be the value of d(E$. $)$ by the composite $L \circ c h$, i. e., $\operatorname{ch}_{X}^{M}(E):.=L \circ \operatorname{ch}(d(E)$.$) . The "twisted" version of$ the localized Chern character is obtained by replacing $c h$ by the Chern character polynomial $c h_{(q)}$. To be more precise, let $\boldsymbol{L}_{(q)}:=\boldsymbol{L} \otimes Q\left[q, q^{-1}\right]: H^{2 *}(M, M-$ $\left.X ; Q\left[q, q^{-1}\right]\right) \rightarrow H_{2 *}\left(X ; Q\left[q, q^{-1}\right]\right)$ be the extension of the Lefschetz duality isomorphism $L$ with respect to the Laurent polynomial ring $Q\left[q, q^{-1}\right]$, and let $c h_{(q)}: K^{0}(M, M-X) \rightarrow H^{2 *}\left(M, M-X ; Q\left[q, q^{-1}\right]\right)$ be the "Chern character polynomial". Then the localized Chern character polynomial (or localized "twisted" Chern character) $\operatorname{ch}_{(q) X}^{M}(E$. $)$ of a complex $E$. is defined by :

$$
\text { Definition (3.5). } \quad \operatorname{ch}_{(q) X}^{M}(E .):=L_{(q)} \circ \operatorname{ch}_{(q)}(d(E .)) \text {. }
$$

Then it is not hard to see that by definition the localized "twisted" Chern character $\operatorname{ch}_{(q) X}^{M}(E$.$) satisfy all the properties (listed in [1, Chap. 1, §§2-3]) enjoyed$ by the localized Chern character $\operatorname{ch}_{X}^{M}(E$.$) , except [1, Chap. 1, Proposition$ (3. 4)]. Namely, in the formulae described in [1, Chap. 1, §§2-3] symbol ch is just replaced by symbol $c h_{(q)}$. Our "twisted" vesion of [1, Chap. 1, Proposition (3.4)] is as follows :

Proposition (3.6). Let $E$. be a complex of bundles on $M$ exact off $X$, and let $\pi: N \rightarrow M$ be a vector bundle over $M$ with $M$ regarded as a subspace of $N$ by the zero-section. Let $\Lambda^{\vee} \pi^{*} N^{\vee}$ be the Koszul-Thom complex on $N$. Then $\Lambda^{*} \pi^{*} N^{\vee} \otimes$ 
$\pi^{*} E$. is exact on $N-X$ and

$$
\operatorname{ch}_{(q) X}{ }^{M}\left(\Lambda^{*} \pi^{*} N^{\vee} \otimes \pi^{*} E .\right)=q^{\mathrm{rank} N}\left(t d_{(q)}(N)^{-1} \cap \operatorname{ch} \underset{(q) X}{M}(E .)\right) .
$$

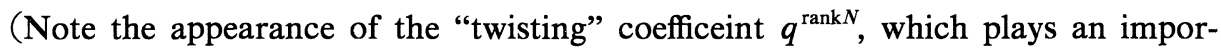
tant role later. Also note that $t d_{(q)}(N)^{-1}$ actually means $i^{*} t d_{(q)}(N)^{-1}$, where $i$ : $X \rightarrow M$ is the inclusion map. However, we sometimes omit the pull-back symbol, unless some confusion occurs.)

Following the proof of [1, Chap. 1, Proposition (3.4)], we can see that the above formula follows from Formula $(3.2)^{(\mathrm{q})}$ in the previous section, so its proof is omitted.

The localized Chern character $c h_{X}^{M}(\mathscr{F})$ of a coherent sheaf $\mathscr{F}$ on $X$ is defined via a resolution $E$. of the coherent sheaf $i_{*} \mathscr{F}$ on $M$, i. e., $\operatorname{ch}_{X}^{M}(\mathscr{F}):=$ $\operatorname{ch}_{X}^{M}(E$.$) . In [1$, Chap. 1] it is shown that it is independent of the choice of resolution $E$. of $i_{*} \mathscr{F}$. When it comes to the twisted verision, one might be tempted to define the "twisted" localized Chen character $\operatorname{ch}_{(q) X}{ }^{M}(\mathscr{F})$ of a coherent sheaf $\mathscr{F}$ on $X$ simply by $\operatorname{ch}_{(q) X}{ }^{M}(E$.). But it is not the case, and our "twisted" version is taken up to be the following :

$$
\text { Definition (3. 7). } \quad \begin{aligned}
\operatorname{ch}_{(q) X}^{M}(\mathscr{F}): & =q^{\operatorname{dim} X-\operatorname{dim} M} \operatorname{ch}_{(q) X}^{M}(E .) \\
(\text { i.e., } & \left.:=q^{\text {reldm }(\imath)} \operatorname{ch}_{(q) X}^{M}(E .)\right) .
\end{aligned}
$$

Of course, this "twisted" localized Chern character of a coherent sheaf $\mathscr{F}$ is independent of the choice of resolution $E$. and if $0 \rightarrow \mathscr{F}^{\prime} \rightarrow \mathscr{F} \rightarrow \mathscr{F}^{\prime \prime} \rightarrow 0$ is a short exact sequence of coherent sheaves on $X$, then $\operatorname{ch}_{(q) X}{ }^{M}(\mathscr{F})=\operatorname{ch}_{(q) X}{ }^{M}\left(\mathscr{F}^{\prime}\right)+$ $\operatorname{ch}_{(q) X} M\left(\mathscr{F}^{\prime \prime}\right)$.

With this definition, it turns out that our "twisted" version of [1, Chap. 1 , Proposition (5. 3), p. 115] is of the same form ; simply "ch" and " $t d$ " being replaced by " $c h_{(q)}$ " and " $t d_{(q)}$ ". Namely, we have

Proposition (3. 8). Let $X \subset M \subset P$ with $M$ and $P$ non-singular quasi-projective varieties. Let $N$ be the normal bundle of $M$ in $P$. Then for any coherent sheaf $\mathscr{F}$ on $X$,

$$
\operatorname{ch}_{(q) X}^{P}(\mathscr{F})=t d_{(q)}(N)^{-1} \cap \operatorname{ch}_{(q) X}^{M}(\mathscr{F}) .
$$

Proof. Let $i: X \rightarrow M$ and $j: M \rightarrow P$ be the inclusion maps. Then by the definition we have 


$$
\operatorname{ch}_{(q) X}^{P}(\mathscr{F})=q^{\operatorname{dim} X-\operatorname{dim} P} \operatorname{ch}_{(q) X}^{P}\left(E^{\sim}\right)
$$

where it should be noted that $E^{\sim}$ is a (or any) resolution of the coherent sheaf $j_{*}\left(i_{*} \mathscr{F}\right)$. Then by [1, Chap. 1, Homotopy Property (2.5) and Lemma (5.2)], we can see

$$
\operatorname{ch}_{(q) X}^{P}\left(E^{\sim}\right)=\operatorname{ch}_{(q) X}{ }^{N}\left(E^{\sharp}\right) .
$$

Here $E^{\sharp}$ is a resolution of $s_{*}\left(i_{*} \mathscr{F}\right)$, where $s: M \rightarrow N$ is the zero-section of the normal bundle $N$. Noticing the fact that the definition of the localized ("twisted") Chern character of a coherent sheaf is independent of the choice of the resolution, and by observing the fact that if we let $E$. be a resolution of $i_{*} \mathscr{F}$, then $\Lambda^{*} \pi^{*} N^{\vee} \otimes \pi^{*} E$. is a resolution of $s_{*}\left(i_{*} \mathscr{F}\right)$, we can see

$$
\operatorname{ch}_{(q) X}^{N}\left(E^{\sharp}\right)=\operatorname{ch}_{(q) X}^{N}\left(\Lambda^{*} \pi^{*} N^{\vee} \otimes \pi^{*} E .\right) .
$$

Hence, by (3. 8. 1-3), we get

$$
\begin{aligned}
& \operatorname{ch}_{(q) X}^{P}(\mathscr{F})=q^{\operatorname{dim} X-\operatorname{dim} P} \operatorname{ch}_{(q) X}^{N}\left(\Lambda^{*} \pi^{*} N^{\vee} \otimes \pi^{*} E .\right) . \\
&=q^{\operatorname{dim} X-\operatorname{dim} P}\left(q^{\text {rankN }} t d_{(q)}(N)^{-1} \cap \operatorname{ch}_{(q) X}^{M}(E .)\right) \\
& \text { (by Proposition (3.6)). }
\end{aligned}
$$

By noticing that $\operatorname{dim} P=\operatorname{dim} M+\operatorname{rank} N$, from (3. 8.4) we get

$$
\operatorname{ch}_{(q) X}^{P}(\mathscr{F})=q^{\operatorname{dim} X-\operatorname{dim} M}\left(t d{ }_{(q)}(N)^{-1} \cap \operatorname{ch}_{(q) X}{ }^{M}(E .)\right) .
$$

Thus by the definition of $\operatorname{ch}_{(q) X}{ }_{M}^{M}(\mathscr{F})$ we get

$$
\operatorname{ch}_{(q) X}^{P}(\mathscr{F})=t d_{(q)}(N)^{-1} \cap \operatorname{ch}_{(q) X}^{M}(\mathscr{F}) .
$$

Q.E. D.

Now we are ready to define our "twisted" version $T d_{(q)} *$ of BFM-Riemann -Roch transformation. For each variety $X$ with $X \subset M, M$ non-singular, the homomorphism $T d_{(q) *}^{M}: K_{0}^{(q)}(X) \rightarrow H_{2 *}^{(q)}(X ; Q)$ is defined as follows : for each coherent sheaf $\mathscr{F}$ on $X$

$$
\operatorname{Td}_{(q) *}^{M}(\mathscr{F}):=t d_{(q)}\left(T_{M}\right) \cap \operatorname{ch}_{(q) X}^{M}(\mathscr{F})
$$

and extend this linearly with respect to $Q\left[q, q^{-1}\right]$.

This definition seems to be dependent on the choice of an embedding $i: X \rightarrow$ 
$M$, but it turns out to be independent of it :

Proposition (3.9). Let $X \subset P$ and $X \subset Q$ be two embeddings of $X$ into nonsingular quasi-projective varieties $P$ and $Q$. Then we have

$$
T d_{(q) *}^{P}=T d_{(q) *} \underset{*}{Q}
$$

i. e., $T d_{(q) *}^{\stackrel{P}{*}}(\mathscr{F})=T d_{(q) \stackrel{Q}{*}}^{\stackrel{Q}{F}}(\mathscr{F})$ for each $\mathscr{F}$.

Proof. First we show the following lemma

Lemma (3.9.1). Let $X \subset M \subset P$, where $M$ and $P$ are non-singular. Then

$$
T d_{(q) *}^{P}=T d_{(q) *}^{M},
$$

i. e., $T d_{(q) *}^{P}(\mathscr{F})=T d_{(q) *}^{M}(\mathscr{F})$ for each $\mathscr{F}$.

Proof of Lemma (3.9.1). Let $i: X \rightarrow M$ and $j: M \rightarrow P$ be the inclusion maps. Then by the definition of $T d_{(q) *}^{P}(\mathscr{F})$, we have

$$
\begin{aligned}
& T d_{(q) *}^{P}(\mathscr{F})= i^{*} j^{*} t d_{(q)}\left(T_{P}\right) \cap \operatorname{ch}_{(q) X}^{P}(\mathscr{F}) \\
&= i^{*}\left(j^{*} t d_{(q)}\left(T_{P}\right)\right) \cap i^{*} t d_{(q)}(N)^{-1} \cap \operatorname{ch}_{(q) X}^{M}(\mathscr{F}) \\
& \quad(\operatorname{by} \text { Proposition }(3.8)) \\
&=i^{*}\left(j^{*} t d_{(q)}\left(T_{P}\right) t d_{(q)}(N)^{-1}\right) \cap \operatorname{ch}_{(q) X}^{M}(\mathscr{F}) \\
&=i^{*}\left(t d_{(q)}\left(\left.T_{P}\right|_{M}\right) t d_{(q)}(N)^{-1}\right) \cap \operatorname{ch}_{(q) X}^{M}(\mathscr{F}) .
\end{aligned}
$$

Since $\left.0 \rightarrow T_{M} \rightarrow T_{P}\right|_{M} \rightarrow N \rightarrow 0$ is exact and $t d_{(q)}$ is multiplicative, we get

$$
t d_{(q)}\left(\left.T_{P}\right|_{M}\right) t d_{(q)}(N)^{-1}=t d_{(q)}\left(T_{M}\right)
$$

Thus we have

$$
T d_{(q) *}^{P}(\mathscr{F})=i^{*} t d_{(q)}\left(T_{M}\right) \cap \operatorname{ch}_{(q) X}^{M}(\mathscr{F})=T d_{(q) *}^{M}(\mathscr{F}) .
$$

Q. E. D.

To finish the proof of the proposition, we consider the embedding $P \subset P \times Q$ by means of the mapping $x \mapsto(x, q)$ for some point $q$ of $Q$, fixed. Similarly we consider the embedding $Q \subset P \times Q$ by means of the mapping $x \mapsto(p, x)$ for some point $p$ of $P$, fixed. Then we have the situation where $X \subset P \subset P \times Q$ and $X \subset Q \subset$ 
$P \times Q$. Hence by Lemma (3.9.1) we have

$$
T d_{(q) *}^{P}(\mathscr{F})=T d_{(q) *}^{P \times Q}(\mathscr{F})=T d_{(q) *}^{Q}(\mathscr{F}) .
$$

Q.E. D.

Thus, for each complex projective variety $X$ the above homomorphism $T d_{(q) *}{ }^{M}: K_{0}^{(q)}(X) \rightarrow H_{2 *}^{(q)}(X ; Q)$ is independent of the choice of an embedding of $X$ into a non-singular quasi--projective variety. (Hence, it suffices to consider the ambient projective space where a given projective varitiey $X$ lies in.) So we can just denote $T d_{(q) *}$ without the superscript.

Now we are ready to state our main theorem :

Theorem (3.10). The transformation $T d_{(q) *}: \mathbb{K}_{0}^{(q)} \rightarrow H_{2 *}^{(q)}(; Q)$ is the unique natural transformation satisfying the extra condition that if $X$ is smooth then $T d_{(q) *}\left(\mathcal{O}_{X}\right)=t d_{(q)}\left(T_{X}\right) \cap[X]$. If we "evaluate" $T d_{(q) *}$ at $q=1$, then we get $B F M-R R$ transformation $T d_{(1) *}=T d_{*}$.

The second statement is clear by the construction of $T d_{(q) *}$ and it is also easy to see (i) that by the construction of $T d_{(q) *}$ the transformation $T d_{(q) *}$ satisfies the above extra condition and (ii) that by Lemma (2.1) $T d_{(q) *}$ is the unique transformation satisfying the above extra condition. Thus it remains to show the naturality of transformation $T d_{(q) *}$, i. e., for any morphism $f: X \rightarrow Y$ the following diagram is commutative :

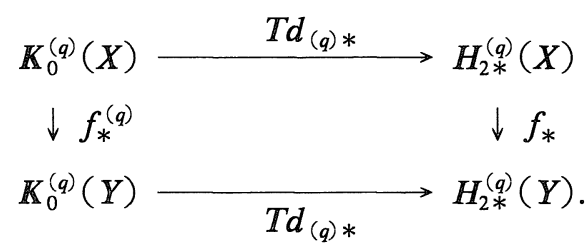

Since any morphism $f: X \rightarrow Y$ is the composite of an imbedding $j: X \rightarrow Y \times$ $\mathbb{P}^{N}$ (for some projective space $\mathbb{P}^{N}$ ) and the projection $\pi: Y \times \mathbb{P}^{N} \rightarrow Y$, i. e., $f=$ $\pi \circ j$, it suffices to show the commutativity of the above diagram for the embedding case when $f: X \rightarrow Y$ is assumed to be an embedding and the projection $\pi: Y \times \mathbb{P}^{N} \rightarrow Y$.

Proposition (3.11). If $f: X \rightarrow Y$ is an inclusion map, then the above diagram is commutative.

Proof. Let $k: Y \rightarrow M$ be an inclusion map, where $M$ is non-singular. It suffices to show that for each coherent sheaf $\mathscr{F}$ 


$$
\begin{aligned}
& f_{*}\left(T d_{(q) *}^{M}(\mathscr{F})\right)=T d_{(q) *}^{M}\left(f_{*}^{(q)}(\mathscr{F})\right) . \\
& f_{*}\left(T d_{(q) *}^{M}(\mathscr{F})\right)=f_{*}\left(f^{*} k^{*} t d_{(q)}\left(T_{M}\right) \cap \operatorname{ch}_{(q) X}^{M}(\mathscr{F})\right) \\
& \text { (by the definition of } T d_{(q) *}^{M}(\mathscr{F}) \text { ) } \\
& =k^{*} t d_{(q)}\left(T_{M}\right) \cap f_{*} c h_{(q) X}^{M}(\mathscr{F}) \text { (by the projection formula) } \\
& =q^{\operatorname{dim} X-\operatorname{dim} M}\left(k^{*} t d_{(q)}\left(T_{M}\right) \cap f_{*} c h_{(q) X}^{M}(E .)\right) \\
& \text { (by the definition of } \operatorname{ch}_{(q) X}(\mathscr{F}) \text { ) } \\
& \left.=q^{\mathrm{d} \mathrm{m} X-\mathrm{d} m M}\left(k^{*} t d_{(q)}\left(T_{M}\right) \cap \operatorname{ch}_{(q)}\right)_{Y}^{M}(E .)\right) \\
& \text { (by }[1, \text { Property (2.1) (a), p. 109]) } \\
& =q^{\operatorname{dim} X-\operatorname{dim} Y}\left(k^{*} t d_{(q)}\left(T_{M}\right) \cap q^{\operatorname{dim} Y-\operatorname{dim} M} \operatorname{ch}_{(q) Y}^{M}(E .)\right) .
\end{aligned}
$$

Here we observe that since $E$. is a resolution of $k_{*} f_{*} \mathscr{F}=k_{*}\left(f_{*} \mathscr{F}\right)$, we have

$$
\operatorname{ch}_{(q) Y}^{M}\left(f_{*} \mathscr{F}\right)=q^{\operatorname{dim} Y-\operatorname{dim} M} \operatorname{ch}_{(q) Y}^{M}(E .) .
$$

Therefore we get that

$$
f_{*}\left(T d_{(q) *}^{M}(\mathscr{F})\right)=q^{\operatorname{dim} X-\operatorname{dim} Y}\left(k^{*} t d_{(q)}\left(T_{M}\right) \cap \operatorname{ch}_{(q) Y}^{M}\left(f_{*} \mathscr{F}\right)\right) .
$$

Since $f_{*}^{(q)}(\mathscr{F})=q^{\text {reldim }(f)} f_{*}(\mathscr{F})=q^{\operatorname{dim} X-\operatorname{dim} Y} f_{*}(\mathscr{F})$ by the definition of our "twisted" pushforward $f_{*}^{(q)}$, we get

$$
\begin{aligned}
f_{*}\left(T d_{(q) *}^{M}(\mathscr{F})\right) & \left.=k^{*} t_{(q)}\left(T_{M}\right) \cap \operatorname{ch}_{(q) Y}^{M}\left(f_{*}^{(q)} \mathscr{F}\right)\right) \\
& =T d_{(q) *}^{M}\left(f_{*}^{(q)}(\mathscr{F})\right) .
\end{aligned}
$$

Q. E. D.

Proposition (3.12). Let $\pi: Y \times P^{N} \rightarrow Y$ be projection. Then the following diagram is commutative :

$$
\begin{array}{ccc}
\boldsymbol{K}_{0}^{(q)}\left(Y \times \boldsymbol{P}^{N}\right) & T d_{(q) *} & H_{2 *}^{(q)}\left(Y \times P^{N}\right) \\
\downarrow \pi_{*}^{(q)} & & \downarrow \pi_{*} \\
\boldsymbol{K}_{0}^{(q)}(Y) & & H_{2 *}^{(q)}(Y) .
\end{array}
$$

Proof. This "projection" case is reduced to showing the commutativity of the above diagram for $Y=$ a point $\{p t\}$ (see [4, p. 287] for the explanation for 
this.) Let $\pi: \mathbb{P}^{N} \rightarrow p t$. Then, since $\boldsymbol{K}_{0}^{(q)}\left(\mathbb{P}^{N}\right)$ is generated by $\mathcal{O}(n)$ and $T d_{(q) *}(\mathscr{F})=i^{*} t d_{(q)}\left(T_{M}\right) \cap \operatorname{ch}_{(q) X}^{M}(\mathscr{F})=t d_{(q)}\left(T_{X}\right) \cap \operatorname{ch}_{(q)}(\mathscr{F}) \cap[X]$ if $X$ is smooth, what we want to show is :

$$
\begin{aligned}
{\left[c h_{(q)}\left(\pi_{*}^{(q)} \mathcal{O}(n)\right) t d_{(q)}\left(T_{\langle p t\}}\right)\right] \cap[p t] } \\
\quad=\pi_{*}\left(\left[c h_{(q)}(\mathcal{O}(n)) t d_{(q)}\left(T_{P^{N}}\right)\right] \cap\left[\mathbb{P}^{N}\right]\right),
\end{aligned}
$$

i. e., by the definition of the twisted pushforward $\pi_{*}^{(q)}$

$$
q^{N} \operatorname{ch}_{(q)}\left(\pi_{*} \mathcal{O}(n)\right)=\int_{P^{N}}\left(\operatorname{ch}_{(q)}(\mathcal{O}(n)) t d_{(q)}\left(T_{P^{N}}\right)\right) .
$$

Here, following Fulton's book [4, Remark 3.2.2.] $\int_{X} \alpha$ denotes the 0-dimensional component of $\alpha \cap[X]$. Since $\pi_{*} \mathcal{O}(n)=\Sigma(-1)^{i} H^{i}\left(\mathbb{P}^{N}, \mathcal{O}(n)\right)$ and $c h_{(q)}$ $=c h_{0}+\Sigma_{i \geq 1} q^{i} c h_{i}$,

$$
\begin{aligned}
q^{N} h_{(q)}\left(\pi_{*} \mathcal{O}(n)\right) & =q^{N} c h_{0}\left(\pi_{*} \mathcal{O}(n)\right) \\
& =q^{N} \Sigma(-1)^{\imath} \operatorname{dim} H^{i}\left(\boldsymbol{P}^{N}, \mathcal{O}(n)\right) \\
& =q^{N} \chi\left(\mathbb{P}^{N}, \mathcal{O}(n)\right) .
\end{aligned}
$$

On the other hand, (see [4, Example 15. 1.4])

$$
\begin{aligned}
\int_{P^{N}}\left(c h_{(q)}\right. & \left.(\mathcal{O}(n)) t d_{(q)}\left(\mathbb{T}_{P^{N}}\right)\right) \\
& =\int_{P^{N}}\left\{e^{q x N}\left(q x / 1-e^{-q x}\right)^{N+1}\right\}, \text { where } x=c_{1}\left(\mathcal{O}\left(\mathbb{P}^{N}\right)(1)\right) . \\
& =\left[q^{N} \chi\left(\mathbb{P}^{N}, \mathcal{O}(n)\right) x^{N}\right] \cap\left[\mathbb{P}^{N}\right] \\
& =q^{N} \chi\left(\mathbb{P}^{N}, \mathcal{O}(n)\right) .
\end{aligned}
$$

Thus (3. 12. 1) holds.

Q. E. D.

As a corollary of Theorem (3.10), we can get the twisted version of GRR :

Corollary (3.13) (A "twisted" version of $(G R R)$. For a non-singular projective variety $X$, if we define the homomorphism $\tau^{(q)}: K_{0}^{(q)}(X) \rightarrow H^{2 *(q)}(X ; Q)(:=$ $\left.H^{2 *}\left(X ; Q\left[q, q^{-1}\right]\right)\right)$ by $\tau^{(q)}(\mathscr{F}):=\operatorname{ch}_{(q)}(\mathscr{F}) t d_{(q)}\left(T_{X}\right)$ and extending linearly with respect to $Q\left[q, q^{-1}\right]$, then $\tau^{(q)}$ becomes a natural transformation, $i$. e., for a morphism $f: X \rightarrow Y$ the following equality holds : 


$$
c h_{(q)}\left(f_{*}^{(q)} \mathscr{F}\right) t d_{(q)}\left(T_{Y}\right)=f_{*}\left(c h_{(q)}(\mathscr{F}) t d_{(q)}\left(T_{X}\right)\right)
$$

where $f_{*}: H^{2 *(q)}(X ; Q) \rightarrow H^{2 *(q)}(Y ; Q)$ is the Gysin homomorphism. Namely, the following diagram commutes :

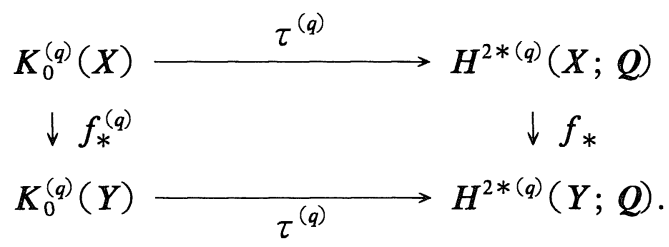

Remark (3.14). Originally we had this theorem first, the proof of which was a "twisted" version of the proof of GRR (see [4, pp. 287-288]), which is left as an exercise for readers. The key point is to see how the twisting coefficient " $q^{\text {some power" }}$ fits in the proof. After we had this twisted version of GRR, we could see how to modify the proof of BFM-RR to get our twisted BFM-RR $T d_{(q) *}$, and then we noticed a relationship between $T d_{(q) *}$ and $T d_{*}$ that for a variety $X$, the homomorphism $T d_{(q) *}: K_{0}^{(q)}(X) \rightarrow H_{2 *}^{(q)}(X ; Q)$ can be simply described as

$$
T d_{(q) *}=q^{\operatorname{dim} X} \sum_{i \geq 0} q^{-i} T d_{* i} .
$$

There are at least two ways to see this. Firstly, if we take a closer look at the definitions $T d_{(q) *}(\mathscr{F})=i^{*} t d_{(q)}\left(T_{M}\right) \cap \operatorname{ch}_{(q) X}(\mathscr{F})$ and $T d_{*}(\mathscr{F})=i^{*} t d\left(T_{M}\right) \cap$ $\operatorname{ch}_{X}^{M}(\mathscr{F})$, in particular $\operatorname{ch}_{(q) X}^{M}(\mathscr{F})$ and $\operatorname{ch}_{X}^{M}(\mathscr{F})$, then by some combinatorial computation (left as an exercise) we can see that

$$
T d_{(q) *}(\mathscr{F})=\sum_{i \geq 0} q^{\operatorname{dim} X-i} T d_{* i}(\mathscr{F})
$$

Secondly, it suffices to show that the transformation $T: K_{0}^{(q)} \rightarrow H_{2 *}^{(q)}(; Q)$ defined by the righthand side of (3.14.1) is a natural transformation satisfying the extra condition that $T\left(\mathcal{O}_{X}\right)=t d_{(q)}\left(T_{X}\right) \cap[X]$ for a smooth $X$. It is easy to see that $T$ satisfies the extra condition. And by the definition of the twisted pushforward $f_{*}^{(q)}$ and the naturality of $T d_{*}$, hence the naturality of $T d_{* i}$, we can see the naturality of $T$. In this sense, Theorem (3.10) itself can be proved very quickly using the natural transformation $T$ defined by the right-hand-side of (3.14.1), but, as stated in the introduction, the aim of our proof of Theorem (3.10) is to show that even if " $t d$ " and " $c h$ " are replaced by " $t d_{(q)}$ " and " $c h_{(q)}$ ", the whole proof of BFM-RR-theorem works by necessarily introducing twisting coefficients " $q$ some power" in suitable places.

Finally, let us call $T d_{(q) *}(X):=T d_{(q) *}\left(\mathcal{O}_{X}\right)$ the twisted homology Todd 
class of $X$. The 0 -th component of $T d_{(q) *}(X)$, denoted by $\chi^{(q)}\left(X, \mathcal{O}_{X}\right)$, is just $q^{\operatorname{dim} X} \chi\left(X, \mathcal{O}_{X}\right)$. Obviously $\chi^{(q)}$ is multiplicative. In fact, $T d_{(q) *}$ is multiplicative. Indeed, using the multiplicativity of the localized twisted Chern character, we can show the following

Proposition (3.15). The followoing diagram is commutative :

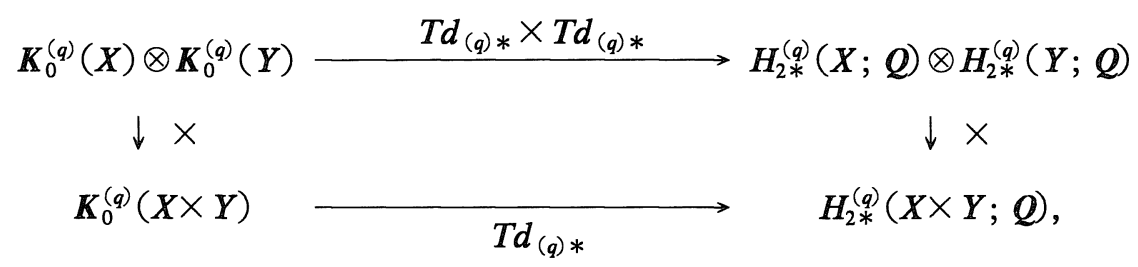

where the vertical homomorphisms are Künneth homomorphisms. In particular,

$$
T d_{(q) *}(X \times Y)=T d_{(q) *}(X) \times T d_{(q) *}(Y) .
$$

This is an analogue of the multiplicativity of the twisted MacPherson classes $[8]$.

Before finishing this section, we give a certain characterization of our twisted version $T d_{(q) *}$ of BFM-RR theorem. Let $\Lambda$ be a field of characteristic zero. Let $c l: \mathscr{V}_{e c t} \rightarrow H^{2 *}(; \Lambda)$ be a total characteristic cohomology class of complex vector bundles. Let $\boldsymbol{K}_{0}^{\Lambda}(\boldsymbol{X}):=\boldsymbol{K}_{0}(\boldsymbol{X}) \otimes \Lambda$.

Definition (3.16). For a morphism $f: X \rightarrow Y$, the pushforward $f_{*}^{c l}: K_{0}^{\Lambda}(X)$ $\rightarrow \boldsymbol{K}_{0}^{\Lambda}(\boldsymbol{Y})$ is defined by

$$
f_{*}^{c l}(\mathscr{F}):=\alpha(f) f_{*}(\mathscr{F})
$$

where the twisting coefficient $\alpha(f) \in \Lambda$ depends only on the characteristic class $c l \operatorname{dim} X$ and $\operatorname{dim} Y$, and $f_{*}$ is the usual pushforward.

If the "twisting" operator $\alpha$, assigning an element $\alpha(f)$ of $\Lambda$ to each morhism $f$, satisfies the property that $\alpha(f \circ g)=\alpha(f) \circ \alpha(g)$, then the above "twisted" pushforward $f_{*}^{c l}$ is functorial. If we consider the above twisted pushforward in the Riemann-Roch type situation, then although we cannot characterize the "twisting" operator $\alpha$ we can characterize the characteristic class $c l$, i. e., we can get the following theorem, which is a sort of characterization of the twisted version $T d_{(q)}$ of $\mathrm{BFM}-\mathrm{RR}$ : 
Theorem (3.17). The twisted pushforward $f_{*}^{c l}$ becomes a covariant functor with a certain twisting operator $\alpha$ so that there exists a (unique) natural transformation $\tau^{c l}: K_{0}^{\Lambda}(X) \rightarrow H_{2 *}(; \Lambda)$ satisfying the extra condition ("smooth clcondition") that $\tau^{c l}\left(\mathcal{O}_{X}\right)=\operatorname{cl}\left(T_{X}\right) \cap[X]$ for any smooth $X$, if and only if $c l=\eta$ $\Sigma_{i \geq 0} \lambda^{i}$ td $_{i}$ for some $\eta$ and $\lambda \in \Lambda$ and $\tau^{c l}=\eta T d_{(\lambda) *}$.

Proof. (If part) Suppose that $c l=\eta \sum_{i \geq 0} \lambda^{i} t d_{i}$ for some $\eta$ and $\lambda \in \Lambda$ and let the transformation $\tau^{c l}:=\eta T d_{(\lambda) *}$. Then, as in the twisted version $T d_{(q) *}$, we just define $f_{*}^{c l}(\mathscr{F}):=f_{*}^{(\lambda)}(\mathscr{F})=\lambda^{\text {reldim }(f)} f_{*}(\mathscr{F})$, then $\tau^{c l}$ is natural. Indeed

$$
\begin{aligned}
\tau^{c l} \circ f_{*}^{c l}(\mathscr{F}) & =\eta T d_{(\lambda) *} \circ f_{*}^{(\lambda)}(\mathscr{F}) \quad \text { (by the definition) } \\
& \left.=\eta f_{*} \circ T d_{(\lambda) *}(\mathscr{F}) \quad \text { (by the twisted version } T d_{(q) *}\right) \\
& =f_{*} \circ\left(\eta T d_{(\lambda) *}(\mathscr{F})\right) \\
& =f_{*} \circ \tau^{c l}(\mathscr{F}) .
\end{aligned}
$$

(Only if part) First, by a similar manner to that of Proof II of Theorem (2.6) (or see [12, Proof of Lemma (2.3)]) we can show that the total characteristic class $c l$ must be a linear form of individual Todd classes, i. e., $c l=\sum_{i \geq 0} \lambda_{i} t d_{i}$ for some $\lambda_{i} \in \Lambda$. From this we can claim in a similar manner to that of $[12$, Theorem (2.2)] that $c l=\eta \sum_{i \geq 0} \lambda^{i}{ }^{i} d_{l}$ for some $\eta$ and $\lambda \in \Lambda$. Hence, by the "smooth cl-condition" we get $\tau^{c l}=\eta T d_{(\lambda) *}$.

Q. E. D.

\section{References}

[1] Baum, P., Fulton, W. and MacPherson, R., Riemann-Roch for Singular Varieties, Publ. Math. IHES., 45(1975), 101-145.

[2] , Riemann-Roch and Topological K-theory for Singular Varieties, Acta Math., 143 (1979), 155-192.

[3] Fulton, W., Rational Equivalence on Singular Varieties, Publ. Math. IHES., 45(1975), 147167.

[4] _ Intersection Theory, Ergebnisse der Mathematik, 3. Folge. 2, Springer-Verlag, 1984.

[5] Gonzalez-Sprinberg, G., L'obstruction locale d'Euler et le théorème de MacPherson, Astérisque, 82-83(1978-1979), 7-32.

[6] Hirzebruch, F., Topological Methods in Algebraic Geometry (3rd ed), Springer-Verlag, 1966.

[7] Kwieciński, M., Formule du produit pour les classes charactéristiques de Chern-SchwartzMacPherson et homologie d'intersection, C. R. Acad. Sci. Paris, 314, Serie I(1992), 625-628.

[8] and Yokura, S., Product formula for twisted MacPherson classes, Proc. Japan Acad., 68(1992), 167-171.

[9] MacPherson, R., Chern classes for singular algebraic varieties, Ann. of Math., 100 (1974), $423-432$. 
[10] MacPherson, R., Characteristic classes for singular varieties, Proc. of the Ninth Brazilian Mathematical Colloquium, II, Instituto de Matematica Pura e Aplicada, São Paulo, (1977), 321-327.

[11] Milnor, J. W. and Stasheff, J. D., Characteristic Classes, Ann. of Math. Studies 76, Princeton Univ. Press, 1974.

[12] Yokura, S., An extension of Deligne-Grothendieck-MacPherson' theory $C_{*}$ of Chern classes for singular algebraic varieties, Publ. RIMS, Kyoto Univ., 27(1991), 745-762.

[13] __ Some variants of Deligne-Grothendieck-MacPherson's natural transformation $C_{*}$ of Chern class, J. für die Reine und Angew. Math.(Crelles J.), 419(1991), 199-211.

[14] A note on the linear independence of Chern numbers and Pontryagin numbers, Math. Japon., 37(1992), 1011-1013.

[15] _ On the universality of Baum-Fulton-MacPherson's Riemann-Roch for singular varieties, Proc. Japan Acad, 68(1992), 119-122. 